\title{
miR-143-3p inhibition promotes neuronal survival in an Alzheimer's disease cell model by targeting neuregulin-1
}

\author{
Chengyan Sun ${ }^{1}$, Ning Jia ${ }^{1}$, Rui Li ${ }^{2}$, Ze Zhang ${ }^{3}$, Yi Zhong ${ }^{3}$, Kun Han ${ }^{1}$ \\ ${ }^{1}$ Department of Neurology, Hwa Mei Hospital, University of Chinese Academy of Sciences, Ningbo Institute of Life and Health \\ Industry, University of Chinese Academy of Sciences, Ningbo, Zhejiang, PR China, ${ }^{2}$ Department of Cardiovascular Medicine, The \\ Third Affiliated Hospital of Jinzhou Medical University, Jinzhou, Liaoning, PR China, ${ }^{3}$ Department of Neurology, The First Affiliated \\ Hospital of Jinzhou Medical University, Jinzhou, Liaoning, PR China
}

\begin{abstract}
Introduction: Alzheimer's disease $(A D)$ is still the fifth leading cause of death and most common dementia worldwide. To date, there is no efficient strategy that can slow down the progression of $A D$ owing to delayed diagnosis and limited therapies. MiR-143-3p is up-regulated in serum of AD patients, yet the exact role it plays in AD pathology is still poorly understood. The aim of this study was to investigate the effect of miR-143-3p on neuronal survival.

Material and methods: We induced neuronal differentiation in SH-SY5Y cells using all-trans-retinoic acid (RA), and A 1-42 was used to establish the in vitro AD cell model. The expression of tubulin $\beta$ III and neuregulin-1 (NRG1) was evaluated by immunofluorescence. TUNEL assay was performed to assess cell apoptosis. Cell viability was evaluated using the Cell Counting Kit-8 assay. The binding interaction between miR-143-3p and NRG1 was verified using the luciferase reporter assay.

Results: Typical neuronal-like axons were observed in RA-induced SH-SY5Y cells, followed by increased tubulin $\beta$ III. A dramatically increased apoptotic rate and reduced cell viability were observed in the AD cell model. Then we silenced the miR-143-3p expression, and AB1-42 induced cell apoptosis was alleviated after miR-143-3p inhibition, accompanied by decreased cleaved caspase-3 and cleaved caspase-9 levels. Additionally, NRG1 was confirmed to be a downstream target of miR-143-3p, increased cell viability and suppressed cell apoptosis after miR-143-3p inhibition was abolished by NRG1 knockdown.

Conclusions: Our findings reveal that miR-143-3p inhibition promotes neuronal survival in an in vitro cell model via targeting NRG1, and the miR-143-3p/NRG1 axis is a potential therapeutic target and promising biomarker for $A D$ treatment.
\end{abstract}

Key words: Alzheimer's disease, neuronal survival, miR-143-3p, NRG1.

\section{Introduction}

Alzheimer's disease (AD) is a type of neurodegenerative disease mainly characterized by memory and cognitive impairment, accompanied by deteriorated behavior and mood [20]. As the most com- mon form of dementia, $A D$ affected approximately 35.6 million people worldwide in 2010 and the number is anticipated to double every twenty years to 115.4 million by 2050, and caring for AD patients is a substantial Medicare cost and a great burden for 
society [26]. Advanced age is the most predominant risk factor for $A D$ as $A D$ cases occur most frequently in patients older than 65 at a risk rate of $10.5 \%$, and incidence rises with age [13]. The major pathogenesis of $A D$ is the deposition of $\beta$-amyloid (AB) peptide cleaved from the amyloid precursor protein, Tau protein hyperphosphorylation and necrosis of neurons [29]. There are only five drug treatments approved for $A D$, which can merely control the clinical symptoms [4]. AD has an insidious onset and patients remain asymptomatic at the early stage of the disease, and most patients are diagnosed as the clinical symptoms emerge, which leave very limited treatment options, let alone provide a cure [11] According to the diagnostic guideline for $A D$ from the National Institute on Aging Alzheimer's Association, the pathophysiological process of $A D$ starts 10 years or even earlier before diagnosis, which makes early detection and intervention at preclinical phases critical [27]. The A $\beta$ level in cerebrospinal fluid (CSF) appears to decrease 25 years before clinical symptoms. $A \beta$ accumulation and an elevated tau protein level were detected 15 years prior to symptom onset, followed by neurodegeneration and cognitive decline [3]. Consequently, it is feasible to find eligible biomarkers for early diagnosis and progression prediction for AD.

MicroRNAs are a class of noncoding transcripts approximately 22 nucleotides in length. MicroRNAs are involved in numerous physiological processes via complementary binding to target sites to downregulate gene expression [14]. MiR-143-3p is significantly up-regulated in the serum of AD patients with mild cognitive impairment, indicating that miR-143$3 p$ might be implicated in the progression of $A D$ [8]. Also, miR-143-3p is elevated in both acute ischemic stroke patients and in a mouse stroke model. Inhibition of miR-143-3p expression can alleviate neurological deficits as well as blood-brain barrier damage in mice [2]. MiR-143-3p silencing also prevents nitric oxide-induced neuroblastoma cell apoptosis and protects neurons from ischemic brain injury in rats $[34,35]$. All these previous findings reveal that miR-143-3p inhibition presents a protective effect on neuronal cells, yet the precise underlying mechanisms remain undiscovered.

Neuregulin-1 (NRG1) is a member of the neuregulin family containing an epidermal growth factor-like domain. NRG1 plays a crucial role in the development of the brain, and it is also expressed in adult brain [31].
NRG1 was also reported to be involved in neurodevelopmental processes including neuronal migration and differentiation as well as synapse formation [5]. Recent studies showed that NRG1 is significantly down-regulated in the hippocampus of AD patients, and NRG1 alleviated cognitive impairment and neuropathology in an AD mouse model [18,32]. Furthermore, NRG1 was a putative downstream target of miR-143-3p by bioinformatics prediction in TargetScan. Therefore, we speculate that miR-143-3p is involved in the development of $A D$ through regulating NRG 1 .

In the present study, human neuroblastoma SH-SY5Y cells were treated with A $\beta 1-42$ to imitate the neuronal injury in AD. We investigated the effect of miR-143-3p on viability and apoptosis in SH-SY5Y cells. We also assessed the interaction between miR143-3p and NRG1.

\section{Material and methods \\ Cell culture, differentiation, transfection, and $A \beta 1-42$ treatment}

SH-SY5Y cells were obtained from Procell (China) and cultured with MEM/F12 culture medium (Procell, China) containing 10\% fetal bovine serum (FBS) (Biological Industries, Israel) in an incubator at $37^{\circ} \mathrm{C}$. Cell differentiation was induced by $10 \mu \mathrm{M}$ all-trans-retinoic acid (RA) (Aladdin, China) in MEM/ F12 culture medium with $1 \%$ FBS for 7 days, and culture medium was replaced every 3 days. Morphological changes in SH-SY5Y cells after RA treatment were observed under a microscope (200x magnification), and differentiated cells were used for all subsequent investigations. SH-SY5Y cells were transfected with microRNAs, microRNA inhibitors or siRNAs using Lipofectamin3000 (Invitrogen, USA) according to the manufacturers' protocol. To establish the in vitro $A D$ cell model, A 1 1-42 (GenScript, USA) was dissolved in pre-cooled hexafluoroisopropanol for 10 minutes, and hexafluoroisopropanol was allowed to volatilize to form an A 1-42 protein membrane, followed by dissolving in DMSO. SH-SY5Y cells were transfected and treated with $1 \mu \mathrm{M} \mathrm{A \beta 1-42}$ for 24 hours to perform the subsequent experiments.

\section{Immunofluorescence and TdT-mediated dUTP nick-end labeling (TUNEL) assay}

For immunofluorescence assay, SH-SY5Y cell slides were fixed with $4 \%$ paraformaldehyde for 15 minutes and permeated with $0.1 \%$ tritonX-100 
for 30 minutes at room temperature. Cell slides were then washed with PBS three times and blocked with goat serum for 15 minutes, followed by incubation with mouse NRG1 antibody (1 : 50, Proteintech, China) or rabbit tubulin $\beta$ III antibody ( $1: 50$, BOSTED, China) overnight at $4^{\circ} \mathrm{C}$. Cell slides were washed with PBS buffer three times and incubated with cy3-labeled goat anti-mouse IgG (1 : 200, Proteintech, China) or cy3-labeled goat anti-rabbit IgG (1 : 200, Proteintech, China) for 60 minutes at room temperature. For TUNEL assay, cell slides were permeated with $0.1 \%$ tritonX-100 and cell apoptosis was detected using a commercial In Situ Cell Death Detection Kit (Roche, Switzerland). After washing with PBS buffer, cell slides were stained with DAPI (Beyotime, China) and sealed with anti-fluorescence quenching reagent (Solarbio, China). Typical images were captured under a microscope (400× magnification).

\section{Cell Counting Kit- 8 assay}

SH-SY5Y cells were seeded onto a 96-well plate $\left(3 \times 10^{3}\right.$ cells/well) and cultured with $100 \mu \mathrm{l}$ of culture medium containing $10 \mu \mathrm{l}$ of CCK-8 (Sigma, USA) for one hour. Optical density values at $450 \mathrm{~nm}$ were recorded and analyzed.

\section{Quantitative real-time PCR}

SH-SY5Y cells were lysed and total RNAs were extracted using a commercial RNA isolation kit (Bioteke, China). Total RNAs were reverse transcribed into cDNA and quantified using SYBR Green (Bioteke, China). $\beta$-actin and small nuclear RNA U6 were used as an internal control. Stem-loop RT primers and real-time PCR primers used in this study are listed below:

Hsa-miR-143-3p specific stem-loop primer: 5'-GTTGGCTCTGGTGCAGGGTCCGAGGTATTCGCACCAGAGCCAACGAGCTA-3';

Hsa-U6 specific stem-loop primer: 5'-GTTGGCTCTG GTGCAGGGTCCGAGGTATTCGCACCAGAGCCAACAAAATATGG-3';

Hsa-miR-143-3p-F: 5'-GAGAGATGAAGCACTGTAGC-3'; Hsa-miR-143-3p-R: 5'-GCAGGGTCCGAGGTATTC-3';

Hsa-U6-F: 5'-GCTTCGGCAGCACATATACT-3'; HsaU6-R: 5'-GTGCAGGGTCCGAGGTATTC-3';

NRG1-F: 5'-CGAAAGCCACTCTGTAATC-3'; NRG1-R: 5'-GTCTCGGTAGGAATCAGG-3';

$\beta$-actin-F: 5'-CACTGTGCCCATCTACGAGG-3'; $\beta$-actin-R: 5'-TAATGTCACGCACGATTTCC-3'.

\section{Western blot}

SH-SY5Y cells were lysed with RIPA buffer (Beyotime, China) and fractionated on SDS polyacrylamide gels. Then total proteins were transferred onto PVDF membranes (Thermo Fisher, China) and blocked with 5\% nonfat milk. PVDF membranes were incubated with primary antibodies overnight at $4^{\circ} \mathrm{C}$, rinsed with PBS buffer three times and incubated with secondary antibodies for 40 minutes at room temperature. Primary antibodies used were NRG1 antibody (1 : 500, Abclonal, China), pro-caspase-3/cleaved caspase-3 antibody (1 : 500, CST, USA), pro-caspase-9/cleaved caspase- 9 antibody ( 1 : 500, CST, USA) and $\beta$-actin antibody (1 : 2000, Proteintech, China). Secondary antibodies used were HRP-conjugated goat anti-rabbit IgG and HRP-conjugated goat anti-mouse IgG (1 : 10000, Proteintech, China). Target protein bands were visualized using ECL reagent (Beyotime, China) and analyzed using Gel-Pro-Analyzer.

\section{Luciferase reporter assay}

A luciferase reporter assay was performed to verify the interaction between miR-143-3p and NRG1. Wildtype and mutant type of NRG1 3'-UTR containing a binding site, termed as NRG1-WT and NRG1-MUT, were amplified and cloned into a pmirGLO plasmid (Promega, USA). HEK-293T cells were seeded onto a 12-well plate and co-transfected with pmirGLO plasmids and NC/miR-143-3p mimics. Luciferase activity was assessed using a commercial Firefly Luciferase Assay Kit (Promega, China) following the manufacturer's instructions.

\section{Statistical analysis}

All data were analyzed using the GraphPad Prism 7 software. The results were displayed as means \pm SD. Student's unpaired $t$-test was used for determining the differences between two groups and for comparisons of more than two groups one-way ANOVA followed by Tukey's multiple comparison test was used. A statistically significant difference in this study was adopted as $p<0.05$.

\section{Results}

\section{Retinoic acid treatment successfully induced SH-SY5Y cell differentiation}

SH-SY5Y cells were treated with RA for 7 days and typical neuronal-like axons were observed under 
A
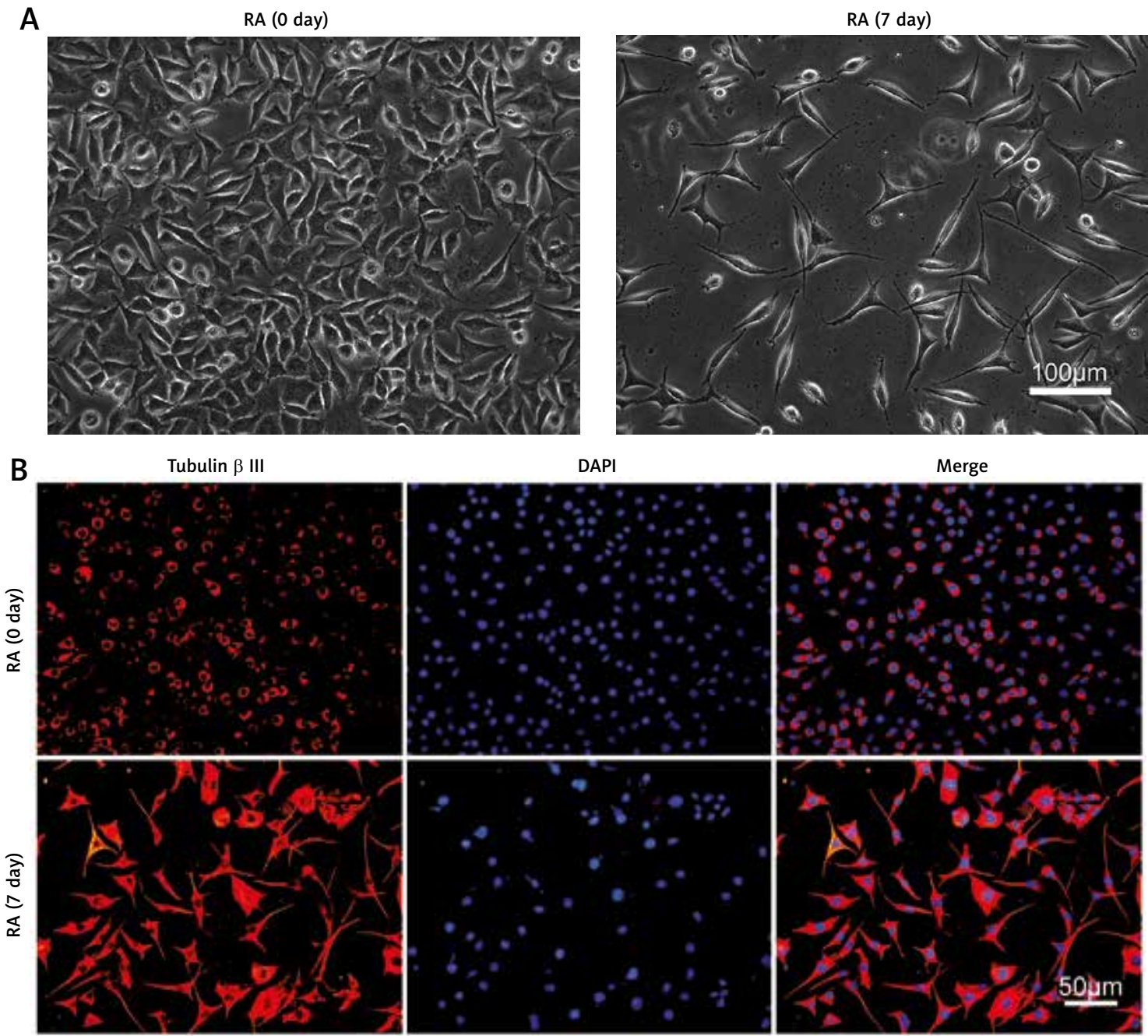

Fig. 1. RA treatment successfully induced SH-SY5Y cell differentiation. SH-SY5Y cells were treated with $10 \mu M$ RA for 7 days. A) Morphological change of SH-SY5Y was observed under a microscope 7 days after RA treatment. B) Expression of tubulin $\beta$ III was assessed by immunofluorescence assay 7 days after RA treatment. RA - retinoic acid.

a microscope (Fig. 1A). Immunofluorescence assay showed that the expression of tubulin $\beta$ III was dramatically increased after RA treatment (Fig. 1B). These results indicated that SH-SY5Y cells were successfully differentiated into neuronal-like cells after RA treatment and these cells were used in all subsequent detections.

\section{In vitro Alzheimer's disease cell model was successfully established}

SH-SY5Y cells were treated with A $\beta 1-42$ and obvious cell apoptosis was detected after treatment (Fig. $2 \mathrm{~A}, \mathrm{~B})$. A $\beta 1-42$ also significantly reduced cell viability in SH-SY5Y cells (Fig. $2 \mathrm{C}$ ), indicating that an in vitro $A D$ cell model was established. Also, the expression level of miR-143-3p was remarkably increased in the AD cell model while the protein level of NRG1 was significantly reduced (Fig. 2D-F).

\section{MiR-143-3p inhibition promoted viability and suppressed apoptosis in SH-SY5Y cells}

SH-SY5Y cells were transfected with specific miR-143-3p inhibitor and treated with A $A 1-42$. $A \beta 1-42$ treatment significantly induced apoptosis in SH-SY5Y cells, which was partially reversed by miR143-3p inhibitor (Fig. 3A, B). A $31-42$ treatment inhibited cell viability in SH-SY5Y cells while further miR-143-3p inhibition abolished the effect (Fig. 3C). 

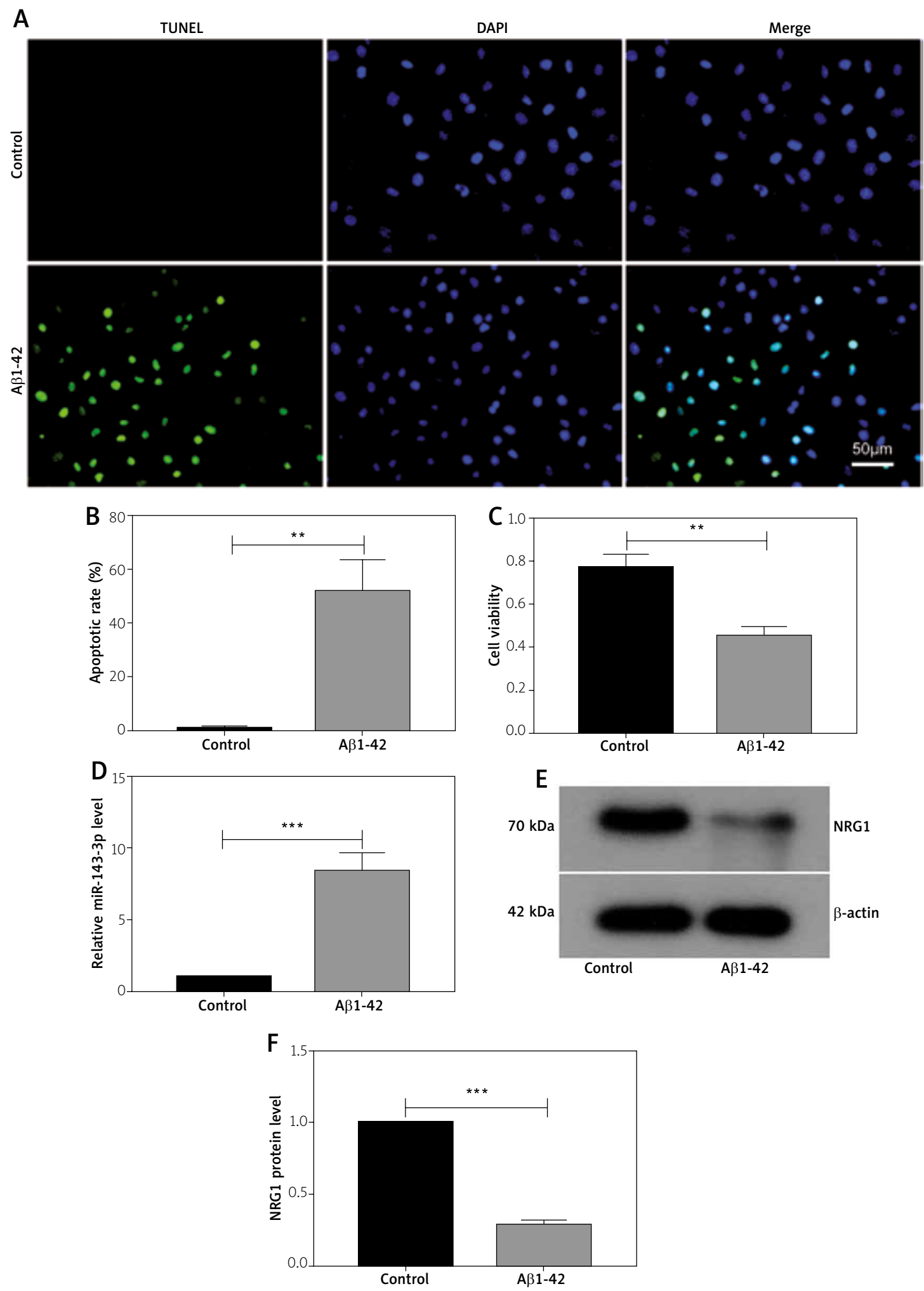

Fig. 2. In vitro AD model was successfully established. SH-SY5Y cells were treated with $10 \mu \mathrm{M} A \beta 1-42$ for 24 hours. A, B) Cell apoptosis was evaluated with TUNEL assay and apoptosis rate was analyzed. C) Cell viability was assessed using commercial CCK-8. D) The miR-143-3p level was measured by quantitative real-time PCR. E, F) The protein level of NRG1 was determined by western blot. AD - Alzheimer's disease; TUNEL - TdT-mediated dUTP nick-end labeling; A $\beta 1-42$ - amyloid- $\beta$ peptide 1-42; CCK-8 - Cell Counting Kit-8; NRG1 - neuregulin $1 ;{ }^{* *} p<0.01 ;{ }^{* * *} p<0.001$. 

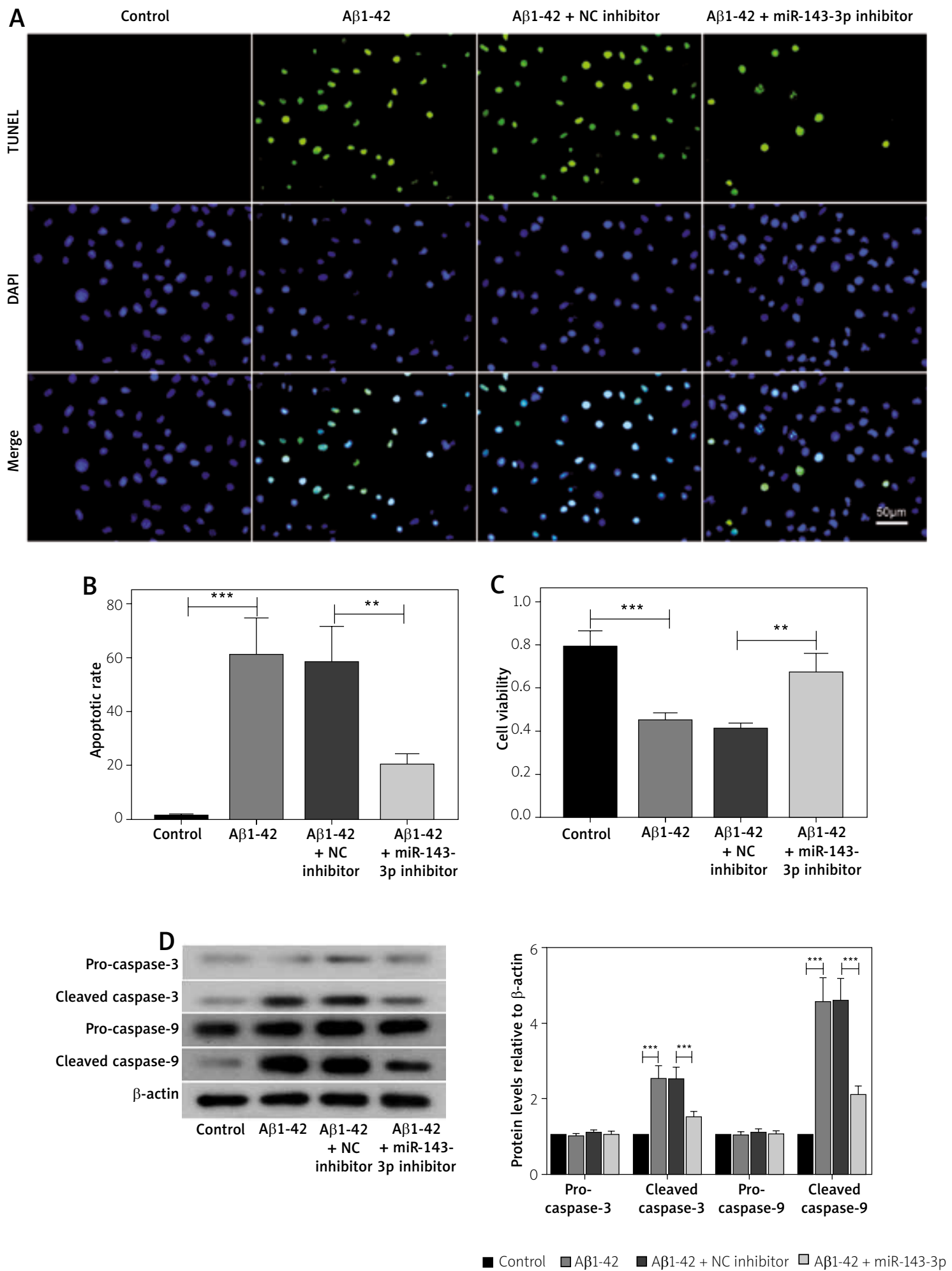

Fig. 3. Effects of miR-143-3p inhibition on viability and apoptosis in SH-SY5Y cells. SH-SY5Y cells were transfected with miR-143-3p inhibitor or non-specific control inhibitor for 24 hours and treated with A $\beta 1-42$ for 24 hours. A, B) TUNEL assay was performed to estimate cell apoptosis. C) Cell viability was assessed by CCK-8 assay. D) Protein levels of pro-caspase-3, cleaved caspase-3, pro-caspase- 9 and cleaved caspase-9 were determined by western blot. ${ }^{* *} p<0.01 ;{ }^{* * *} p<0.001$. 

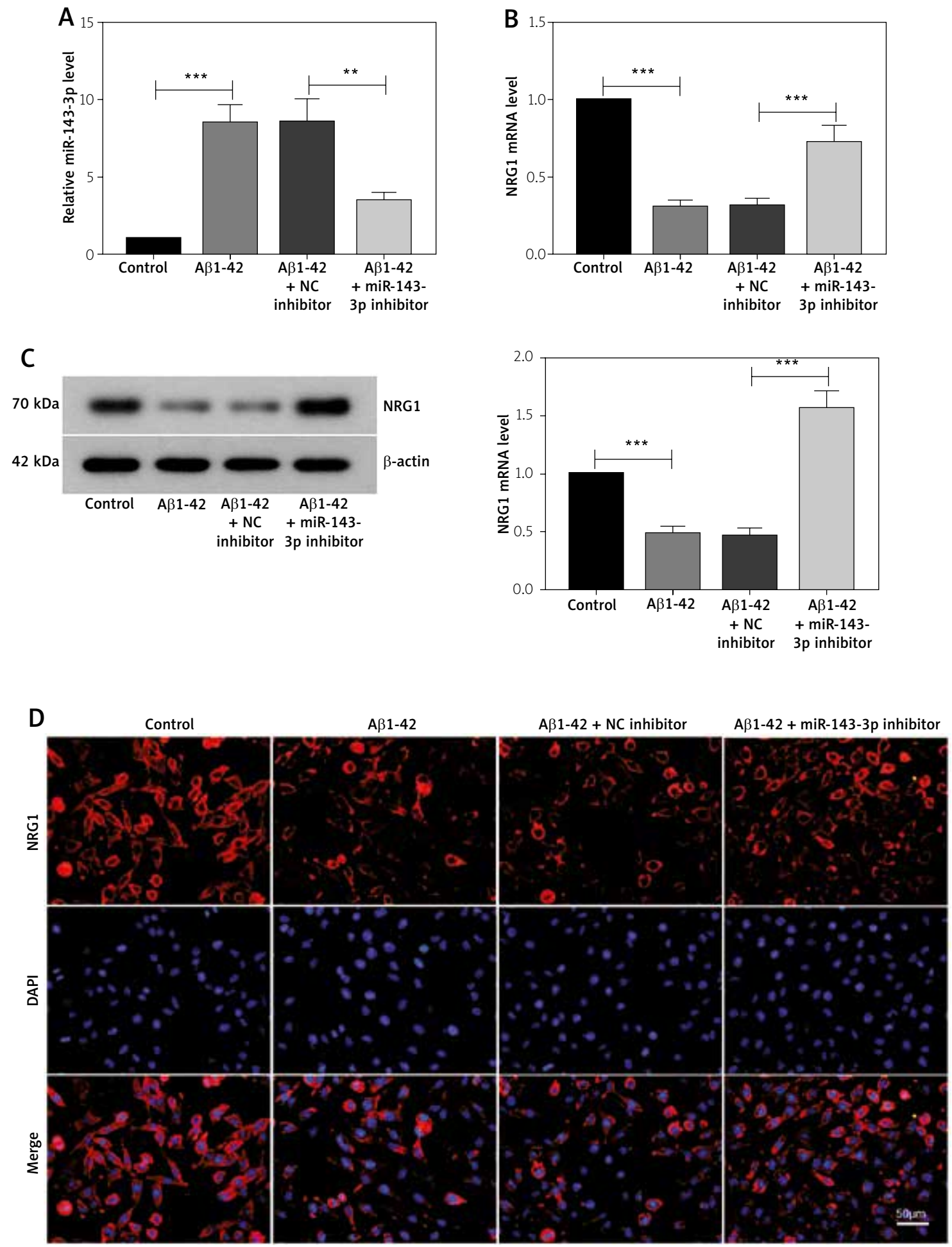

Fig. 4. NRG1 expression was up-regulated after miR-143-3p inhibition in AD cell model. SH-SY5Y cells were transfected with miR-143-3p inhibitor or non-specific control inhibitor for 24 hours and treated with A 1-42 for 24 hours. A, B) Expression levels of miR-143-3p and NRG1 were measured using quantitative real-time PCR. C) The protein level of NRG1 was determined by western blot. D) Localization and expression of NRG1 were detected by immunofluorescence assay, ${ }^{\star *} p<0.01 ;{ }^{* \star *} p<0.001$. 
A

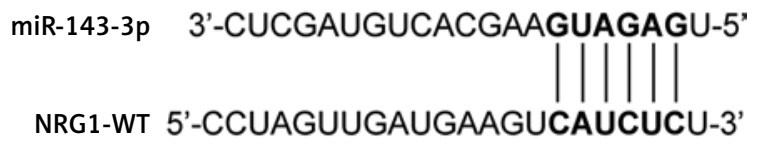

NRG1-MUT 5'-CCUAGUUGAUGAAGUGUAGAGU-3'

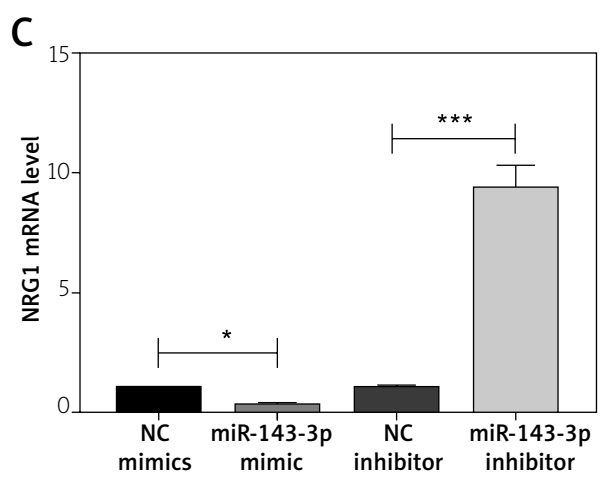

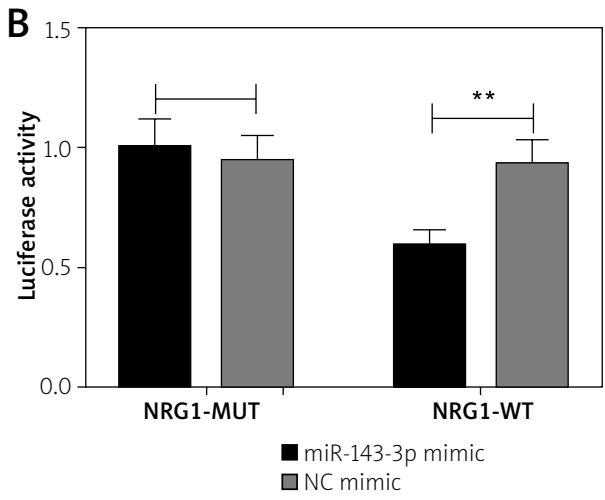

D

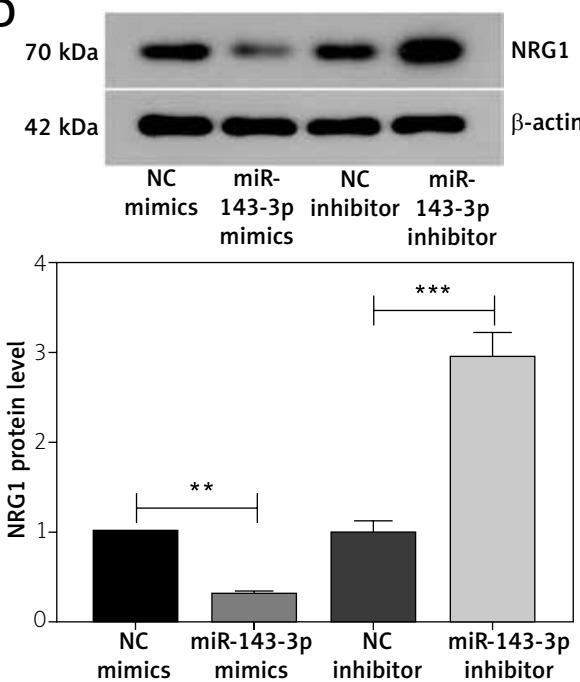

Fig. 5. NRG1 was a downstream target of miR-143-3p. A, B) Wildtype and mutant type of NRG1 3'-UTR containing binding site of miR-143-3p were co-transfected with NC/miR-143-3p mimics into HEK-293 cells. Binding site was shown, and interaction between miR-143-3p and NRG1 was verified using luciferase reporter assay. C, D) SH-SY5Y cells were transfected with NC/miR-143-3p mimics or NC/miR-143-3p inhibitor. Expression level of NRG1 was determined using quantitative real-time PCR and western blot, ${ }^{*} p<0.05$; ${ }^{* *} p<0.01 ;{ }^{* *} p<0.001$.

Also, the protein levels of cleaved caspase- 3 and cleaved caspase-9 were elevated in SH-SY5Y cells after Aß1-42 treatment while further miR-143-3p inhibition reversed the effect (Fig. 3D). These results indicated that miR-143-3p inhibition promoted viability and suppressed apoptosis in the AD cell model.

\section{NRG1 expression was up-regulated after miR-143-3p inhibition in AD cell model}

The expression of miR-143-3p was significantly elevated in the AD cell model, which was partially abrogated after miR-143-3p inhibition (Fig. 4A). NRG1 expression was reduced after $A \beta 1-42$ treatment, which was reversed by further miR-143-3p inhibition at both mRNA and protein levels (Fig. 4B, C). A similar trend was observed in the immunofluorescence assay, and NRG1 was mainly localized in the cytoplasm (Fig. 4D).

\section{NRG1 was a downstream target of miR-143-3p}

The binding site of miR-143-3p on NRG1 is shown in Figure 5A. MiR-143-3p mimics significantly suppressed the luciferase activity in NRG1-WT yet had no obvious effect on NRG1-MUT compared to NC mimics (Fig. 5B). Also, miR-143-3p mimics inhibited NRG1 expression while miR-143-3p inhibitor 

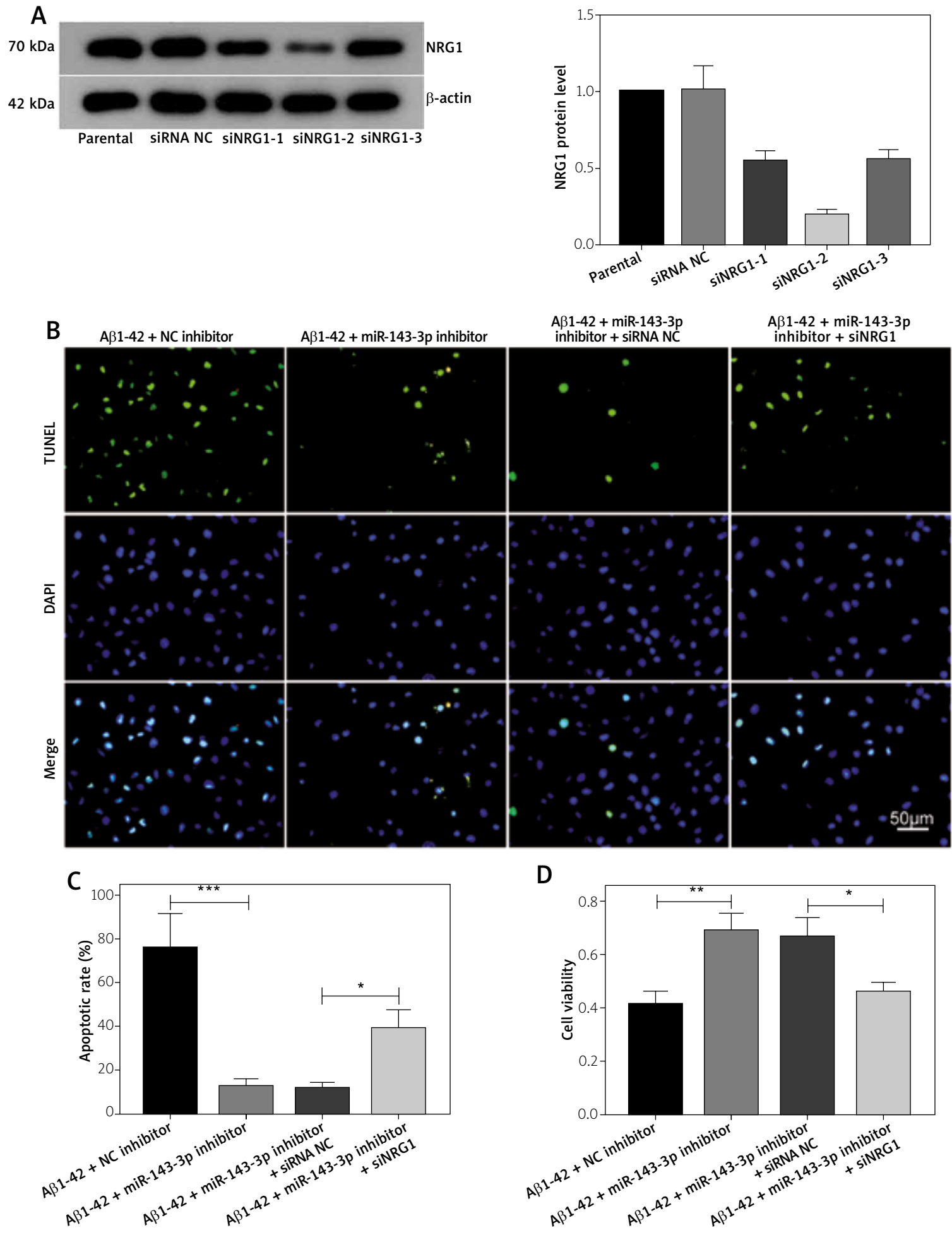

Fig. 6. MiR-143-3p regulated apoptosis and viability in SH-SY5Y cells via targeting NRG1. SH-SY5Y cells were co-transfected with NRG1 siRNA and miR-143-3p inhibitor for 24 hours, and treated with $10 \mu M$ A $31-42$ for 24 hours. A) SH-SY5Y cells were transfected with NRG1 siRNAs and NRG1 protein level was determined by western blot. B, C) Cell apoptosis was evaluated using immunofluorescence assay and apoptosis rate was calculated. D) CCK-8 assay was performed to evaluate cell viability, ${ }^{*} p<0.05 ;{ }^{* *} p<0.01 ;{ }^{* *} p<0.001$. 
elevated NRG1 expression at both mRNA and protein levels (Fig. 5C, D). These results indicated that NRG1 acted as a downstream target of miR-143-3p.

\section{MiR-143-3p regulated apoptosis and viability in SH-SY5Y cells via targeting NRG1}

We silenced NRG1 expression using three specific siRNAs, among which siNRG1-2 showed the highest efficiency and was used for subsequent investigations (Fig. 6A). MiR-143-3p inhibitor alleviated A 1-42-induced cell apoptosis, which was further abrogated after siNRG1 transfection (Fig. 6B, C). Also, miR-143-3p inhibitor promoted cell viability in A 1-42-treated SH-SY5Y cells, and the effect was abolished by further NRG1 silencing (Fig. 6D). These results indicated that miR-143-3p inhibition suppressed apoptosis and promoted viability in $\mathrm{SH}-\mathrm{SY} 5 \mathrm{Y}$ cells via targeting NRG1.

\section{Discussion}

Despite great efforts made in investigating the etiology of $A D, A D$ remains the fifth leading cause of death around the world [36]. Accumulated evidence has shown that $A \beta$ plays a role in $A D$ pathogenesis $[6,21]$. Although the function of $A \beta$ in $A D$ remains controversial, $A \beta$ is still the most well-known causative factor for the disease [33]. In recent years, a variety of cell models have been developed for a better understanding of AD pathogenesis $[12,28]$. Amyloid $\beta$ accumulation is one of the hallmarks of $A D$, among which $A \beta 1-42$ is the most toxic form and widely used in $A D$ in in vitro model induction [25]. Loo et al. [22] established an AD cellular model by adding $A \beta$ to the cell culture medium, and they found that apoptosis may play a role in the neuronal loss associated with AD. Hu et al. [17] suggested that trans-caryophyllene may be able to exert a protective effect against $A \beta$-induced neuroinflammation in AD. Human neuroblastoma cell line SH-SY5Y is widely used in research on neurodegenerative diseases including Parkinson's disease and AD [1]. In the present study, we induced neuronal differentiation of SH-SY5Y using $10 \mu \mathrm{M}$ RA, a vital signaling molecule during neuronal differentiation [23]. Typical neuronal-like morphological features were observed, concomitant with an elevated level of tubulin $\beta$-III, one of the neuron-specific markers [9]. We induced SH-SY5Y with A 42 in our study; reduced cell viabil- ity and increased neuronal apoptosis were detected, indicating that we successfully built an in vitro cell model to mimic AD pathologic condition.

Treatment of $A D$ patients is always delayed owing to lack of efficient early diagnosis, which prevents early intervention [19]. Several microRNAs are emerging as critical regulators for AD pathogenesis including tau protein hyperphosphorylation, neuroinflammation and neuronal apoptosis [10]. Circular RNA DLGAP4 ameliorates neurological deficits and promotes cerebrovascular integrity by sponging miR-143-3p in an ischemic stroke mouse model [2]. MiR-143-3p inhibition also alleviates oxygen-glucose deprivation by targeting the glycolysis enzyme hexokinase 2 and further ameliorates ischemia injury [35]. Furthermore, excessive production of nitric oxide is implicated in various neurodegenerative diseases, including $A D$, by inducing neuronal apoptosis through regulating miR-143-3p [34]. These previous works suggest that miR-143-3p inhibition exerts a neuroprotective effect in neurodegenerative disorders. In the present study, miR-143-3p was up-regulated in an AD cell model, which was highly consistent with the previous study in serum of AD patients [8]. Moreover, miR-143-3p inhibition improved cell viability and reduced cell apoptosis, accompanied by elevated cleaved caspase- 3 and caspase- 9 . A $\beta 42$ induces neuronal apoptosis via activating pro-apoptotic proteins, inducing cytochrome $c$ release and further activating caspases, followed by the mitochondrial mechanism of cell apoptosis [15]. Thus our results indicated that miR-143-3p inhibition alleviated neuronal apoptosis in the AD cell model through the mitochondrial pathway.

NRG1 level was decreased in both AD patients and an in vivo mouse model. NRG1 is imperative for neural and cardiac development. NRG1 functions via specifically binding to the ErbB family of receptor tyrosine kinases, especially ErbB4, which also acts as a susceptibility gene for AD pathology [30]. The activation of NRG1 is based on the $\beta$-site of amyloid precursor protein-cleaving enzyme (BACE1), a crucial enzyme involved in the pathogenesis of AD [24]. BACE1-dependent cleavage of NRG1 is critical for its functions in synaptic plasticity and normal psychiatric behaviors [16]. NRG1 modulates the cell surface expression of amyloid precursor protein (APP), affects the production of $A \beta$ and further hinders the progress of AD [18]. NRG1 can also attenuate cognitive deficits and neuropathology to play a neuronal- 
protective role in an AD animal model, accompanied by a reduced $A \beta$ level [32]. Furthermore, soluble NRG1 was reported to be a potential biomarker for $A D$ diagnosis as there is a significant difference of soluble NRG1 levels between mild AD patients and control subjects [7]. The negatively correlated expression of NRG1 and miR-143-3p inspired us to investigate their interaction in the AD cell model. Furthermore, we proved that NRG1 is a downstream target of miR-143-3p. A $1-42$ treatment inhibited NRG1 expression, while further miR-143-3p inhibition abolished the effect at both mRNA and protein levels. Also, miR-143-3p inhibition suppressed cell apoptosis and improved cell viability, which was further reversed after NRG1 knockdown by specific siRNAs. Our current results revealed that miR-143-3p inhibition has a neuroprotective effect in an AD cell model and alleviates neuronal apoptosis via targeting NRG1. The limitation of our study is the lack of in vivo experiments to confirm the effect of miR-143-3p on $A D$.

In conclusion, we successfully established an in vitro AD cell model. Our findings demonstrated that miR-143-3p regulates neuronal survival through the intrinsic apoptosis pathway by targeting NRG1. The miR-143-3p/NRG1 axis may serve as a potential diagnostic and therapeutic target for AD treatment.

\section{Acknowledgements}

This study was supported by grants from the Guide Project for Natural Science of Liaoning Province (No. 2019-ZD-0616) and the Doctoral Startup Foundation of the Department of Science and Technology, Liaoning Province (No. 201601357).

\section{Disclosure}

The authors report no conflict of interest.

\section{References}

1. Alberio T, Lopiano L, Fasano M. Cellular models to investigate biochemical pathways in Parkinson's disease. FEBS I 2012; 279: 1146-1155.

2. Bai Y, Zhang Y, Han B, Yang L, Chen X, Huang R, Wu F, Chao J, Liu P, Hu G, Zhang JH, Yao H. Circular RNA DLGAP4 ameliorates ischemic stroke outcomes by targeting miR-143 to regulate endothelial-mesenchymal transition associated with blood brain barrier integrity. J Neurosci 2018; 38: 32-50.

3. Bateman RJ, Xiong C, Benzinger TL, Fagan AM, Goate A, Fox NC Marcus DS, Cairns NJ, Xie X, Blazey TM, Holtzman DM, Santacruz A, Buckles V, Oliver A, Moulder K, Aisen PS, Ghetti B,
Klunk WE, McDade E, Martins RN, Masters CL, Mayeux R, Ringman JM, Rossor MN, Schofield PR, Sperling RA, Salloway S, Morris JC. Clinical and biomarker changes in dominantly inherited Alzheimer's disease. N Engl J Med 2012; 367: 795-804.

4. Briggs R, Kennelly SP, O'Neill D. Drug treatments in Alzheimer's disease. Clin Med (Lond) 2016; 16: 247-253.

5. Buonanno A, Fischbach GD. Neuregulin and ErbB receptor signaling pathways in the nervous system. Curr Opin Neurobiol 2001; 11: 287-296.

6. Carrell RW, Lomas DA. Conformational disease. Lancet (London) 1997; 350: 134-138.

7. Chang KA, Shin KY, Nam E, Lee YB, Moon C, Suh SH, Lee SH. Plasma soluble neuregulin-1 as a diagnostic biomarker for Alzheimer's disease. Neurochem Int 2016; 97: 1-7.

8. Cheng L, Doecke JD, Sharples RA, Villemagne VL, Fowler CJ, Rembach A, Martins RN, Rowe CC, Macaulay SL, Masters CL, Hill AF. Prognostic serum miRNA biomarkers associated with Alzheimer's disease shows concordance with neuropsychological and neuroimaging assessment. Mol Psychiatry 2015; 20: 1188-1196.

9. Cheung YT, Lau WK, Yu MS, Lai CS, Yeung SC, So KF, Chang RC. Effects of all-trans-retinoic acid on human SH-SY5Y neuroblastoma as in vitro model in neurotoxicity research. Neurotoxicology 2009; 30: 127-135.

10. Dehghani R, Rahmani F, Rezaei N. MicroRNA in Alzheimer's disease revisited: implications for major neuropathological mechanisms. Rev Neurosci 2018; 29: 161-182.

11. DeKosky ST, Marek K. Looking backward to move forward: early detection of neurodegenerative disorders. Science 2003; 302: 830-834.

12. Dezfulian M. A new Alzheimer's disease cell model using B cells to induce beta amyloid plaque formation and increase TNF alpha expression. Int Immunopharmacol 2018; 59: 106-112.

13. Guerreiro R, Bras J. The age factor in Alzheimer's disease. Genome Med 2015; 7: 106.

14. He L, Hannon GJ. MicroRNAs: small RNAs with a big role in gene regulation. Nature Rev Genet 2004; 5: 522-531.

15. Hikisz P, Ilianska ZM. PUMA, a critical mediator of cell death - one decade on from its discovery. Cell Mol Biol Lett 2012; 17: 646-669.

16. Hu X, Fan Q, Hou H, Yan R. Neurological dysfunctions associated with altered BACE1-dependent Neuregulin-1 signaling. J Neurochem 2016; 136: 234-249.

17. Hu Y, Zeng Z, Wang B, Guo S. Trans-caryophyllene inhibits amyloid beta (Abeta) oligomer-induced neuroinflammation in BV-2 microglial cells. Int Immunopharmacol 2017; 51: 91-98.

18. Kim YJ, Yoo JY, Kim OS, Kim HB, Ryu J, Kim HS, Lee JH, Yoo HI, Song DY, Baik TK, Woo RS. Neuregulin 1 regulates amyloid precursor protein cell surface expression and non-amyloidogenic processing. J Pharmacol Sci 2018; 137: 146-153.

19. Kuehn B. Alzheimer burden. JAMA 2018; 320: 1851.

20. Kukull WA, Bowen JD. Dementia epidemiology. Med Clin North Am 2002; 86: 573-590.

21. Lee KW, Seomun Y, Kim DH, Park SY, Joo CK. Possible role of amyloid beta-(1-40)-BSA conjugates in transdifferentiation of lens epithelial cells. Yonsei Med J 2004; 45: 219-228.

22. Loo DT, Copani A, Pike CJ, Whittemore ER, Walencewicz AJ, Cotman CW. Apoptosis is induced by beta-amyloid in cultured cen- 
tral nervous system neurons. Proc Natl Acad Sci USA 1993; 90 : 7951-7955.

23. Maden M. Retinoic acid in the development, regeneration and maintenance of the nervous system. Nat Rev Neurosci 2007. 8: 755-765.

24. Mei L, Xiong WC. Neuregulin 1 in neural development, synaptic plasticity and schizophrenia. Nat Rev Neurosci 2008; 9: 437 452.

25. Pahrudin Arrozi A, Shukri SNS, Wan Ngah WZ, Mohd Yusof YA, Ahmad Damanhuri MH, Makpol S. Evaluation of the expression of amyloid precursor protein and the ratio of secreted amyloid beta 42 to amyloid beta 40 in SH-SY5Y cells stably transfected with wild-type, single-mutant and double-mutant forms of the APP gene for the study of Alzheimer's disease pathology. Appl Biochem Biotechnol 2017; 183: 853-866.

26. Prince M, Bryce R, Albanese E, Wimo A, Ribeiro W, Ferri CP. The global prevalence of dementia: a systematic review and metaanalysis. Alzheimers Dement 2013; 9: 63-75.e62.

27. Sperling RA, Aisen PS, Beckett LA, Bennett DA, Craft S, Fagan AM, Iwatsubo T, Jack CR Jr., Kaye J, Montine TJ, Park DC, Reiman EM, Rowe CC, Siemers E, Stern Y, Yaffe K, Carrillo MC, Thies B, Morrison-Bogorad M, Wagster MV, Phelps $\mathrm{CH}$. Toward defining the preclinical stages of Alzheimer's disease: recommendations from the National Institute on Aging-Alzheimer's Association workgroups on diagnostic guidelines for Alzheimer's disease. Alzheimers Dement 2011; 7: 280-292.

28. Stansley B, Post J, Hensley K. A comparative review of cell culture systems for the study of microglial biology in Alzheimer's disease. J Neuroinflammation 2012; 9: 115.

29. Swanberg MM, Tractenberg RE, Mohs R, Thal LJ, Cummings JL. Executive dysfunction in Alzheimer disease. Arch Neurol 2004; 61: 556-560

30. Tian J, Geng F, Gao F, Chen YH, Liu JH, Wu JL, Lan YJ, Zeng YN, Li XW, Yang JM, Gao TM. Down-regulation of neuregulin1/ErbB4 signaling in the hippocampus is critical for learning and memory. Mol Neurobiol 2017; 54: 3976-3987.

31. Woo RS, Li XM, Tao Y, Carpenter-Hyland E, Huang YZ, Weber J, Neiswender H, Dong XP, Wu J, Gassmann M, Lai C, Xiong WC, Gao TM, Mei L. Neuregulin-1 enhances depolarization-induced GABA release. Neuron 2007; 54: 599-610.

32. Xu J, de Winter F, Farrokhi C, Rockenstein E, Mante M, Adame A, Cook J, Jin X, Masliah E, Lee KF. Neuregulin 1 improves cognitive deficits and neuropathology in an Alzheimer's disease model. Sci Rep 2016; 6: 31692.

33. Xu J, Li D, Zheng T, Lu Y. Beta-amyloid expression in age-related cataract lens epithelia and the effect of beta-amyloid on oxidative damage in human lens epithelial cells. Mol Vis 2017; 23: 1015-1028.

34. Yang HJ, Ju F, Guo XX, Ma SP, Wang L, Cheng BF, Zhuang RJ, Zhang $B B$, Shi X, Feng ZW, Wang M. RNA-binding protein RBM3 prevents NO-induced apoptosis in human neuroblastoma cells by modulating p38 signaling and miR-143. Sci Rep 2017; 7: 41738.

35. Zeng X, Liu N, Zhang J, Wang L, Zhang Z, Zhu J, Li Q, Wang Y. Inhibition of miR-143 during ischemia cerebral injury protects neurones through recovery of the hexokinase 2-mediated glucose uptake. Biosci Rep 2017; 37: BSR20170216.
36. Zhou Y, Sun Y, Ma QH, Liu Y. Alzheimer's disease: amyloid-based pathogenesis and potential therapies. Cell Stress 2018; 2: 150161. 